\title{
CHIEF DENTISTS' PERCEIVED STRENGTHS AND WEAKNESSES OF ORAL HEALTH PROMOTION ACTIVITIES IN MALAYSIA
}

N. Ab Murat, R.G. Watt. Chief Dentists' Perceived Strengths and Weaknesses of Oral Health Promotion Activities in Malaysia. Annal Dent Univ Malaya 2006; 13: 1-5.

\begin{abstract}
The main aim of this study is to get the Malaysian chief dentists' perceptions of the oral health promotion activities currently taking place in their respective states in terms of the strengths and weaknesses of these activities. A qualitative method using an open-ended questionnaire was used to obtain this information. The study samples consist of all the chief dentists in Malaysia who represented a majority of population in their states. The results showed that the main current oral health promotion activities is dental health education talks which aims to disseminate oral health information through health education talks, toothbrushing drills, dental exhibition, pamphlets, brochures and publications. Half of the respondents felt that the commitment of the staff, the support of the public sectors and collaboration with other agencies are the main strengths while the lack of staff and fund has been said as the weaknesses. A range of opportunities however exist to strengthen oral health promotion in Malaysia.
\end{abstract}

Key words: oral health promotion, Malaysia.

\section{INTRODUCTION}

The origins of health promotion lie in the $19^{\text {th }}$ century when epidemic disease led to pressure for sanitary reform for the poor and overcrowded living conditions. In 1974, Lalonde described health promotion as the first step in development of a new approach to population health. He expressed the view that the major causes of mortality and morbidity were due to environmental causes, individual behaviours, and lifestyle factors rather than to biomedical characteristics. In 1977, WHO launched Health for All by the Year 2000 which proposed a wider agenda for health linked to socioeconomic change. A later publication, the Ottawa Charter (WHO, 1986) provide the strategic framework that was needed to achieve Health for All (1). It used the terms enabling, mediating and advocating to describe what Health Promotion could achieve. The Ottawa Charter presented 5 keys area which provide a useful structure to explore options for promoting oral health. It tackles the underlying
Original Article

N. Ab Murat ${ }^{1}$, R.G. Watt ${ }^{2}$
${ }^{1}$ Department of Community Dentistry
Faculty of Dentistry
University of Malaya
50603 Kuala Lumpur, Malaysia.
Tel no: 03-79674866 / 012-2840552
Email: norintan@um.edu.my
${ }^{2}$ Professor
Department of Epidemiology and Public Health
UCL Medical School, 1-19 Torrington Place
London WC1E 6EA, United Kingdom.
Corresponding Author - Norintan Ab Murat

determinants of oral health and thereby improves oral health of all.

Health education is one of the strategies within the health promotion policy, but it differs from health promotion. Health education is the process by which people are given the knowledge and awareness through the provision of information so that they will have greater control over their own health. Health education programmes include providing information, exploring values and attitudes, making health decisions and acquiring skills to enable behaviour change to take place. They involve promoting self-esteem and self-empowerment so that people are enabled to take action about their health (2). However, despite the comprehensive programmes in health education, a series of criticisms have been made. The health education approach has been criticized as being too narrowly focussed and is based on the expert authority model derived from both medicine and education. Being expert-led it assumes that the individual has a limited amount of knowledge. People are seen as empty vessels who will rationally change their behaviour once provided with relevant information (3). The health education approach neglects the impact of the environmental and political determinants of health, which might limit the ability of individuals to use this new knowledge.

Health promotion represents a comprehensive social and political process, it not only embraces actions directed at strengthening the skills and capabilities of individuals, but also action directed towards changing social, environmental and economic conditions so as to alleviate their impact on public and individual health (1). Health promotion includes strategies to develop the 
environment so as to make the health promoting choices the easier choices (4). According to Watt et al (5), health promotion has three important elements; focus on tackling the determinants of health, working in partnership and adopting a strategic approach utilizing a complementary range of actions to promote the health of the population.

Health promotion involves the population as a whole in the context of their everyday lives rather than focusing on people who are sick or at risk for specific disease. Actions are addressed towards the many underlying factors influencing health in order to ensure that the environment, which is beyond the control of individuals, is conducive to health. This is based upon the Whole Population Strategy (6). This approach is powerful because a small shift in the population distribution may have a large effect on the number of people affected. Although it is more costly than the High Risk Strategy, its benefits are more long term and it is more appropriate as it tries to change the normal behaviour of the population to a accepted behaviour for good health (6). This approach also recognized the limited control that individuals have over their health and this will prevent victim blaming.

A strategic approach is required for the development of effective health promotion policies. Conventional dental health education is neither effective nor efficient. They always work in isolation from other health programmes which could lead to duplication of effort and conflicting messages given to public (7). Health professionals must use a common risk factor approach when dealing with diseases. This approach addresses risk factors common to many chronic conditions. As dental problem is not considered as a major problem in one's life, by using the common-risk factor approach, people will be more prepared to change their behaviour if they know it will also influence other diseases. In implementing health promotion, planning is important, with evaluation of the strategy as a crucial part.

In order to evaluate the strengths and weaknesses of the oral health promotion activities currently taking place in Malaysia, it was necessary to get the view of the leader of oral health service in every state. This could be best achieved through the collection of qualitative data which would give a valuable insight of the activities. Therefore the aim of this study is to get the Malaysian chief dentists' perceptions of the oral health promotion activities currently taking place in their respective states in terms of the strengths and weaknesses of these activities.

\section{MATERIALS AND METHODS}

This study is a cross-sectional study designed to find out about chief dentists' perceived strengths and weaknesses of the current oral health promotion activities taking place in Malaysia. A qualitative method using an open-ended questionnaire was used to obtain information on the oral health promotion activities and the chief dentists' perceived strengths and weaknesses of these activities.

Fifteen state Chief Dental Officers in Malaysia including two federal territories were invited to participate in this survey. They are chosen as they are considered as one of the key decision maker of oral health programmes in their state. The state chief dentists are based at the capital city in their respective states. So it is important to get their view as their decisions will affect the majority of the population. Although the policy is top down, their knowledge and experience will give an invaluable insight of oral health promotion activities in Malaysia.

The questionnaire was developed by the researchers. Most of the questions are open-ended so as to get a more in depth view from the study sample on the topic.

The Oral Health Division, Ministry of Health was contacted and permission to carry out the survey was obtained. The questionnaire was checked and approved by the head of Chief Dental Officers. A supportive letter signed by the head of chief dentist was sent to all the chief dentists explaining the purpose of the study. The names and addresses of all chief dentists were obtained through the Ministry of Health.

The questionnaire and the cover letter were posted to the chief dentist in the second week of July 2003. The respondents were given one week to reply to the questionnaire. A self-addressed express mail envelope was supplied to each of the chief dentist. Telephone calls were made to those who did not reply within the given time.

Responses to all of the questions were reviewed and analysed to assess common themes. Direct quotations were extracted from the completed questionnaires where appropriate. Due to the limited number of respondents in the study, statistical analysis was not deemed necessary or appropriate.

\section{RESULTS}

\section{Response rate:}

Nine chief dentists replied eventually, giving a response rate of $60 \%$. Thus caution should therefore be exercised in generalising the finding to reflect the views of all state chief dentists. The poor response could be because of the short time frame given to the study sample. They were only given 10 days to answer the questions given and it was commented by one non-respondent to be too detailed and too time consuming to be answered in a very short time. Most of the study samples were not in their office and could not be contacted during the period of the 
study. Several telephone calls were made to them, but most were out of their state attending meetings. Their email addresses could not be obtained so it is impossible to distribute the questionnaire through electronic mail.

\section{Current oral health promotion activities:}

A majority of the respondents reported that the main current oral health promotion activities in their states is dental health education talks which targets the pre-school children, school children and pregnant mothers. One respondent summed up the oral health promotion activities as:

Dissemination of oral health information through health education talks, toothbrushing drills, dental exhibition, pamphlets, brochures and publications.

Respondent 5

Two reported giving dental health education through the radio and another two respondents stated role modelling as one of the activities for primary school children.

It is reported that dental health exhibitions are usually done in schools or in public areas, such as shopping complexes or are done during community programmes. One respondent gave Health Awareness Programmes as one example and another gave the Exhibition of the History of Dentistry that was exhibited in the museum as another example of dental exhibition. The Healthy Lifestyle Campaign is one example of dental campaign currently taking place.

One respondent divided the activities according to target groups:

Medically compromised /disabled people: teaching of cases/parents, hands-on tooth cleaning using volunteer groups

Elderly: instructions on dental care, health talks. Respondent 7

Another respondent conducted joint training session with pre-school and school teachers as a way to increase awareness of the importance of oral health in schools. Other current oral health promotion activities reported taking place in Malaysia included puppet shows, teeth competition and slide show for school children and a specific outreach programme for screening of oral condition.

A majority of the study sample viewed that the activities that they are doing link with the general health promotion activities. Most give the Healthy Lifestyle Campaign and the Health Camp as the example. Two felt that the activities are partly a joint effort and referrals are required from the health service for the toddler's and pregnant mothers programme.
The perceived strengths and weaknesses of these oral health promotion activities:

Half of the respondents felt that the commitment of the staff, the support of the public sector and collaboration with other agencies are the main strengths of oral health promotion activities in their states. One respondent summed up the strengths of oral health promotion in his state as the following:

$$
\begin{aligned}
& \text { - widespread and systematic } \\
& \text { - well incorporated and structured in the } \\
& \text { programme } \\
& \text { - target groups incorporated }
\end{aligned}
$$

Respondent 2

One respondent felt that the strength lies in the dental health education which starts at young age. Another reported that the readiness of the management to respond to requests for oral health promotion activities and the interest shown by external agencies helps to make the activities a success.

As for the weaknesses, almost all respondents expressed that lack of staff and lack of commitment as a major point. There was consensus that the staff involved are neither dedicated nor experienced in handling the activities. One commented on the characteristic of the activities:

It has the tendency to be carried out as a routine and the activities are implemented by the same team each year.

Respondent 2

Two respondents felt that short of personnel create a low frequency of activities. Two respondents also mentioned about the lack of funds and time:

There is not enough dedicated staff for these activities and funds are needed and should be adequate for oral health promotion to be successful.

Respondent 1

Another weakness mentioned by two respondents is the lack of evaluation activities on the effectiveness of oral health promotion activities. One respondent commented that it is a constant battle to change the perception and mindsets of the population who perceive that oral diseases are nonlife threatening condition.

\section{Recommendations and Comments of the chief dentists:}

When asked how they would recommend to improve the quality of oral health promotion activities, six of the respondents recommended the formation of a special team that will only concentrate on delivering oral health promotion activities. As recommended by one respondent: 
A special team should be formed that can implement oral health promotion activities with greater commitment and more innovatively.

Respondent 3

One respondent added that the choice of personnel should be considered:

...only those that are interested and committed should be given the task to ensure the effectiveness of the programme.

Respondent 4

Two respondents recommended continuous training for the personnel in tandem with current concepts and also in graphics and multimedia. One recommended that evaluation of the activities should be done every year while another felt that screening to identify individuals with oral health risks should be done so that counselling can be given on an individual basis. Only one respondent mentioned about getting increased funding for oral health promotion activities as he felt that eventually the cost of treatment may reduce.

Two respondents mentioned about the need to increase collaborative efforts with other sectors and between districts to improve the quality of life:

There should be a partnership with other department and an oral health promotion team should be formed at a state and district level to develop good strategic activities to promote oral health.

Respondent 1

Half of the respondents added some comments on the study topic. One felt that oral health promotion should be on the mass media to enable a wider coverage. Another respondent viewed that the regulation on food labelling should be enforced and there should be a change of focus of oral health care from disease oriented to self-image and quality of life.

One respondent believed that the role of manufacturers is important in promoting health:

Since the manufacturers of consumer products in oral health already play a big part in sponsoring major dental events, they can play a bigger role in educating the public at the same time of promoting their products.

Respondent 7

Finally, one respondent gave a constructive comment in which he believed that oral health promotion activities should:

1) give emphasis on holistic approach in the dissemination of oral health, with integration of oral health messages into other general health messages.

2) emphasis on preventive oral health care approaches and routine dental care examination.

Respondent 6

\section{DISCUSSION}

The main current activity of oral health promotion in Malaysia is dental health education which aims to disseminate information through dental health talks, dental exhibitions, pamphlets and brochures. It is the ethical responsibility of the health professionals to disseminate oral health knowledge to the public, but they and the policy-makers should prepare the ground for other interventions too, such as the creation of more supportive environment.

Messages given during dental health education can be conflicting and confusing. Some groups may emphasise parts of the message at the expense of others. This could undermine the credibility of all those involved in promoting health and makes the public more confuse. If they believed that the experts disagree then the health message quickly loses credibility (8). Health education messages must evolve in the light of new knowledge and policy must be reviewed periodically. In the United Kingdom, the Health Education Authority published in 1996 a document, The Scientific Basis of Dental Health Education, which refined and standardised the advice given to the public. This is to ensure that such advice was scientifically sound. It would be good if the Malaysian oral health professionals and academics could collaborate and produce such a document that is appropriate the local population.

Oral health promotion in Malaysia should try to adopt a more innovative health promotion style of working. Health promotion commit not only to improving lifestyles but also to improving the environment within which lifestyle choices are made. This means that educational activities also need to be targeted at influential individuals whose decisions affect the environment and to enhance the capacity of the population to minimize or eliminate at risk behaviours.

The strengths of oral health interventions has been summed up as widespread and systematic and are well incorporated and structured in the programme. Almost all agreed that collaboration with other agencies has enhanced the oral health promotion activities. The lack of staff and funds has been said as the weaknesses of the oral health strategies.

A strategic approach is required for the development of oral health intervention. By using the Common Risk Factor Approach (7), the partnership and collaboration among agencies can be enhanced. It has been shown that this approach is more effective and efficient and the cost is lower as the burden is shared among the partners. When there are alliances with other sectors, the public can be ensure that the information that they receive are consistent and correct. The scarce resources for oral health promotion is another reason why oral health promoters should adopt this practice. 
There was a recommendation that a special team that will focus only on oral health promotion activities be formed so as to get a greater commitment from the staff. As there are lack of dedicated staff, interest and time in delivering oral health promotion, policy makers in Malaysia should put more thought on this recommendation. In the United Kingdom, there are courses that teach people on how to become oral health educators. The students are not necessarily a dentist or a dental nurse, but they could be anybody who is interested in educating people in oral health. A teacher can be a good example as they already have teaching skills. The courses could include any skill that is required in an oral health promoter. Upon completion of the courses, dental health educators should be active in training other primary health care workers (including dental). Outside the health service they should teach the teachers and key decision makers and community leaders.

Dental health educators should be the one who will make an effort to collaborate with other agencies in any oral health promotion activities. They will be the one who will make sure that the public are getting consistent and correct health messages by dealing and making scientific decisions with other health promoters. There should be a career development for these oral health educators so as to promote people to take up this course. They could become the head of the oral health promotion programme. Another advantages of having an oral health educator is that dentists can start focus on other work such as patient care, personnel management, promoting professional standards and administration including implementing policy; and at the same time ensure that the patient or public are getting the right oral health messages and are able to make healthy choices.

\section{CONCLUSION}

The main current activities of oral health promotion in Malaysia are dental health education where information is disseminated through dental health talks, dental exhibitions, pamphlets and brochures. Through the perceptions of the strengths and weaknesses of these activities by the chief dentists, it can be concluded that there is a need to review options for developing more comprehensive oral health promotion activities in Malaysia.

\section{REFERENCES}

1. World Health Organization (1986). Ottawa Charter for Health Promotion. WHO.

2. Ewles, L. and Simnett, I. (1998). Promoting Health: A Practical Guide, p 88. London: Bailliere Tindall.

3. Naidoo, J. and Wills, J. (1998). Health Promotion: foundations for practice, p 282, London: Bailliere Tindall.

4. Milio, N. (1986). Promoting Health Through Public Policy. Canadian Public Health Association, Ottawa.

5. Watt, R., Fuller, S., Harnett, R., Treasure, E. and Stillman-Lowe, C. (2001). Oral Health Promotion Evaluation- time for development. Community Dent Oral Epidemiol 29: 161-166.

6. Rose, G. (1992). The Strategy of Preventive Medicine. Oxford: Oxford University Press.

7. Sheiham, A. and Watt, R. (2000). The Common Risk Factor Approach: A rational basis for promoting oral health, Community Dent Oral Epidemiol 28, 399-406.

8. Watt, R. and Fuller, S (1999). Oral Health Promotion - opportunity knocks! Br Dent J; 186: 3-6. 